\title{
CCD photometry of late-type stars in the young open cluster IC 2602
}

\author{
D.C. Foster ${ }^{1}$, P.B. Byrne ${ }^{1}$, S.L. Hawley ${ }^{2, \star}$, and W.R.J. Rolleston ${ }^{3}$ \\ 1 Armagh Observatory, College Hill, Armagh BT61 9DG, N. Ireland \\ e-mail: dcf@star.arm.ac.uk, pbb@star.arm.ac.uk \\ 2 Dept. of Physics and Astronomy, Michigan State University, East Lansing, MI 48824-1116, U.S.A. \\ e-mail: slh@pillan.pa.msu.edu \\ ${ }^{3}$ Dept. of Pure and Applied Physics, The Queen's University of Belfast, Belfast BT7 1NN, N. Ireland \\ e-mail: R.Rolleston@Queens-Belfast.ac.uk
}

Received July 7, 1996; accepted February 27, 1997

\begin{abstract}
We present the results of $V R I$ photometry of the young open cluster IC 2602. Two $15 \operatorname{arcmin} \times$ 15 arcmin fields were observed in February and May 1991 using the 1-m Swope telescope at Las Campanas. Using theoretical isochrones obtained from D'Antona \& Mazzitelli (1994), and allowing for observational and other uncertainties, we identify 78 primary candidate members with $12<V<18.5$ from their positions on colourmagnitude diagrams. We compare the cluster field with an offset field of similar galactic latitude and estimate the contamination due to background stars to be large, $\geq 50 \%$, as might be expected given its low galactic latitude. We also compare our photometry with that given for the X-ray detected stars of Randich et al. (1995). We present complimentary narrow band $\mathrm{H} \alpha$ photometry for a subset of the stars.
\end{abstract}

Key words: stars: evolution — stars: late-type — open clusters: individual; IC 2602

\section{Introduction}

Ever since the pioneering work of Wilson (1963) and Kraft (1967), it has generally been accepted that stars of solar mass or less lose their angular momentum with time. Further research by van den Heuvel \& Conti (1971) and Skumanich (1972) suggested that low-mass stars arrive on the main-sequence rotating rapidly, as a consequence of the conservation of angular momentum during the premain-sequence contraction phase. Drawing together both Ca II emission data (indicative of chromospheric activity in late-type stars) and projected rotational velocities

Send offprint requests to: D.C. Foster

* NSF Young Investigator. (used to determine angular momenta) for a small number of stars in three open clusters, viz. Pleiades, Ursa Major and Hyades, and using the solar values, Skumanich derived empirical relationships between the afore-mentioned properties and age, which simply stated that both Ca II H $\& \mathrm{~K}$ emission reversals and stellar rotation declined with time according to an inverse power law. Such relationships were consistent with the theoretical predictions of Durney (1972) based on models in which rotational braking was caused by a stellar wind. This view remained unchallenged for more than a decade.

More recently, new observations have given rise to a new paradigm. Stauffer et al. (1984, 1985, 1987), following up the discovery of rapidly rotating $\mathrm{K}$ stars in the Pleiades (van Leeuwen et al. 1987), measured rotational $v \sin i$ for GKM-type dwarfs in the $\alpha$ Persei (age $\sim 50 \mathrm{Myr}$ ), Pleiades (age $\sim 70 \mathrm{Myr}$ ) and Hyades (age $\sim 800 \mathrm{Myr}$ ) open clusters. These results showed that all late-type members of $\alpha$ Per exhibit a very large spread of rotational $v \sin i$ values, ranging from approximately $15-200 \mathrm{~km} \mathrm{~s}^{-1}$. By contrast, G-type Pleiads had $v \sin i$ close to or less than the observational limit of $10 \mathrm{~km} \mathrm{~s}^{-1}$ with rapid rotation only observed amongst the $\mathrm{K}$ - and M-types.

Inter-comparison of these two clusters suggests that a more rapid braking mechanism than that provided by the classical stellar wind scenario must be at work. This mechanism must be capable of braking the rotation of G-dwarfs on a time-scale of the order of the age difference between $\alpha$ Per and the Pleiades, i.e. $\sim 20$ Myr. Furthermore, only moderately rapid rotation was detected in the oldest of the clusters, the Hyades, and then only in the M-type dwarfs. Comparison of these results supports the idea that, once this rapid phase of braking is complete, a power law relation may then apply.

These important conclusions have been based on the comparison of results found for three open clusters. In 
order to place constraints on possible braking mechanisms, further observations are required of young clusters with ages distributed over the critical range $10-200 \mathrm{Myr}$. Clusters younger than, and of similar age to, $\alpha$ Per are needed to confirm that the rapid braking of G-dwarfs is universal, while those intermediate in age between the Pleiades and Hyades will yield information on the timescales for braking of progressively lower mass stars.

The authors have undertaken such a programme to investigate the distribution of stellar rotation in a number of open clusters. However, as a consequence of the intrinsic faintness of late-type dwarfs $\left(M_{V} \approx 6\right.$ at $\mathrm{K} 0, M_{V} \approx 9$ at $\mathrm{M} 0$ ), it is necessary to restrict the study to clusters that are within approximately $400 \mathrm{pc}$ of the Sun; otherwise the measurement of $v \sin i$ from high-resolution spectroscopy will not be observationally feasible for a sample of their late-type members. Due to their relative proximity, these clusters have a large extent on the sky and unambiguous identification of bona fide members is difficult. With this in mind, we have selected several target clusters for which we have obtained $B V R I$ CCD photometry. Further details of the observational programme and background material can be found in Rolleston (1995).

\section{IC 2602}

The cluster IC $2602\left(\alpha=10^{\mathrm{h}_{43}} \mathrm{~m}_{.0},-64^{\circ} 24^{\prime}, \mathrm{J} 2000.0\right)$ is a group of stars distributed about the B0Vp star $\theta$ Carinae. Early studies (Whiteoak 1961; Braes 1962) determined the cluster to be at a distance of $150-155 \mathrm{pc}$. They estimated the cluster to be approximately 8-12 Myr old, derived both from the nuclear age of $\theta$ Car, and the contraction of cluster members on to the main sequence. More recent studies (Mermilliod 1981) estimate the age to be nearer 36 Myr.

The reddening has been determined to be $E_{B-V}=$ 0.04 (Whiteoak 1961; Braes 1962) corresponding to $E_{V-I}=0.044$ (Randich et al. 1995). Photometric measurements for the brighter stars were obtained (Braes 1962; Whiteoak 1961 and Hill \& Perry 1969), and some of these stars have been studied spectroscopically (Whiteoak 1961; Abt \& Morgan 1972). From measurements of 22 confirmed members, Braes (1962) has estimated the mean cluster proper motion to be $\overline{15 \mu_{\alpha} \cos \delta}=-8.8 \pm$ $1.0 \mathrm{mas} / \mathrm{yr}, \overline{\mu_{\delta}}=3.5 \pm 1.5 \mathrm{mas} / \mathrm{yr}$.

More recently the cluster has been studied in the X-ray wavelength region (Randich et al. 1995). The X-ray data from ROSAT PSPC pointings detected 110 objects, 68 of which were identified with optical counterparts. The study also included CCD photometry in $V$ and $I$ of the central 3.3 square degrees of cluster, with a magnitude limit of $V<18$, and the photometry of the optical counterparts was used to determine the likelihood of the counterparts being cluster members. Additional photometry was published by Prosser et al. (1996). 44 of the X-ray sources were deemed to be possible cluster members. The X-ray luminosity distribution function for the F, G and early $\mathrm{K}$ stars showed the cluster to be more X-ray luminous than the Pleiades. There was little difference for the late $\mathrm{K}$ and M-type stars. Randich et al. argue that, unless their sample is affected by incompleteness, their result is consistent with the younger age of IC 2602 compared with the Pleiades, the younger earlier type stars having had less time to spin down and thereby decrease X-ray luminosity. The later types, having longer braking timescales, would be rapid rotators in both clusters and hence have similar $\mathrm{X}$-ray luminosities.

\section{Observations}

Our photometry was obtained using the 1-m Swope telescope at Las Campanas Observatory on the nights of 9-11 February and 17-18 May 1991. The detector was a Ford2 $2048 \times 2048$ pixel CCD, rebinned $2 \times 2$. At the Cassegrain focus each rebinned pixel corresponds to 0.87 arcsec on the sky. Observations were made through a Johnson $V$ filter, Gunn $r$ and $i$ filters and a narrow band (70 ̊) $\mathrm{H} \alpha$ interference filter.

Two cluster fields were observed. Another field, offset approximately 1 degree from the cluster, but at a similar galactic latitude, was also observed in order that some estimate of the contamination by galactic field stars might be made. The suitability of this field for this purpose is discussed in Sect. 6.2. The coordinates of each of the field centres are given in Table 1 and their positions on the sky are shown in Fig. 1.

For each field, four exposures were made, one short and three long to record respectively the bright and faint stars at optimum exposure levels. Exposure times were $15: 10: 10 \mathrm{~s}$ for the short and $600: 600: 600 \mathrm{~s}$ for the long through $V: R: I$ filters respectively. Three exposures of 1800 seconds each were made of the first cluster field using the $\mathrm{H} \alpha$ filter.

Photometric standard stars chosen from Landolt (1992) to cover a range of spectral types were observed during each night. Dome flat fields for the $V, R$ and $I$ filters, sky flat-fields for the $\mathrm{H} \alpha$ filter, and bias frames were also taken.

Table 1. Coordinates of the field centres

\begin{tabular}{lcccc}
\hline \hline Field & $\alpha$ & $\begin{array}{c}\delta \\
(\mathrm{J} 2000.0)\end{array}$ \\
& \multicolumn{4}{c}{$l$} \\
\hline IC 2602a & $10: 44: 44.4$ & $-64: 29: 04.0$ & 289.792 & -4.924 \\
IC 2602b & $10: 41: 01.5$ & $-64: 11: 27.2$ & 289.328 & -4.802 \\
"Offset" & $10: 35: 15.7$ & $-63: 54: 46.6$ & 288.635 & -4.853 \\
\hline
\end{tabular}




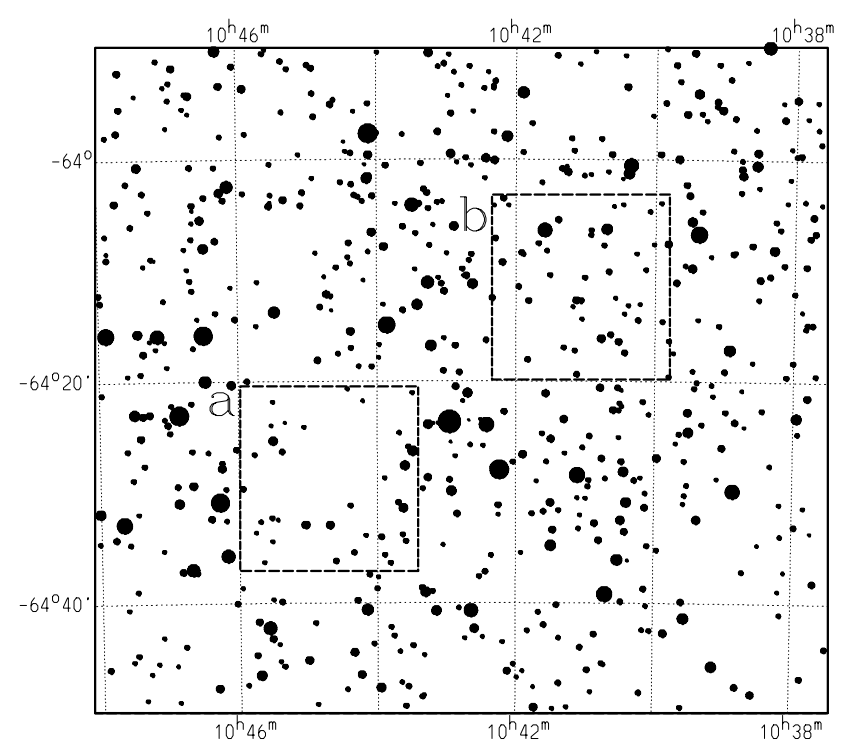

Fig. 1. The region of IC 2602 showing the positions of the two observed cluster fields

\section{Data reduction}

The basic CCD reduction was carried out using IRAF's CCDRED package (Tody 1986). The raw images were trimmed and overscan subtracted. No additional bias subtraction was found necessary. Multiple dome flat-fields (sky flat-fields in the case of the $\mathrm{H} \alpha$ fields) were averaged, and the data flat-fielded using the resultant images. Further details of this process can be found in Massey (1992).

Since the CCD pixel size $(0.87$ arcsec $)$ was large compared to typical seeing conditions encountered at Las Campanas we measured the full-width at half-maximum (FWHM) of a number of moderately exposed stellar images on each frame and found the average value to be close to 2 pixels. Thus we judged the stellar images to be undersampled for determining and applying the pointspread function (PSF) method of photometry needed for the crowded cluster fields.

To overcome this we first used the aperture photometry routines within the DAOPHOT (Stetson 1987; Stetson et al. 1990) package in IRAF for measuring the Landolt standards. An aperture of radius 16 pixels or 14 arcsec was chosen to match Landolt's original aperture as closely as possible. These measurements were used to transform measurements from the instrumental system to standard magnitudes (Johnson-Cousins). The equations used were of the form:

$$
\begin{aligned}
& v=V+k_{v_{1}}+k_{v_{2}} X+k_{v_{3}}(V-R) \\
& r=R+k_{r_{1}}+k_{r_{2}} X+k_{r_{3}}(V-R) \\
& v-i=k_{v-i_{1}}+k_{v-i_{2}} X+k_{v-i_{3}}(V-I)
\end{aligned}
$$

where $v r i$ are the instrumental magnitudes, $V R I$ are the standard magnitudes, $X$ is the airmass and the $k$ are the transformation coefficients. It should be noted that since the reddest observed standard had $V-I=2.268$, there is some uncertainty associated with the colours determined for stars redder than that. Isolated stars were then located on the cluster frames and these were measured with this "standard" aperture and a variety of smaller apertures to determine the dependence of the derived magnitude on aperture size. This we refer to as an "aperture correction".

Because of crowding, particularly a problem for the fainter stars, it was necessary to use PSF photometry on the cluster frames. A best fit PSF was determined and subtracted for each object frame independently. The residuals from the PSF subtraction were found to be mainly in the core of the profile and these were measured using aperture photometry with a radius equal to the FWHM of the PSF. This latter quantity was typically $10 \%$ of the PSF flux itself, independent of magnitude. A PSF correction was accordingly determined for each cluster star individually and applied to the PSF-derived magnitude followed by an "aperture correction" as described above. The accuracy of this method was tested using isolated stars on the object frames and was found to produce errors which were less than those from other sources (see Table 2 below). These instrumental magnitudes were then transformed to the standard system using the transformation equations described above.

The three sets of $V, R, I$ photometry, resulting from the three individual deep CCD exposures in each colour, were then combined in order to eliminate spurious detections resulting from the undersampling (e.g. cosmic rays). Only stars which were common to at least two of the three sets were used. The photometry was then averaged. $77 \%$ of the stars thus selected had a range in measured $V$ magnitude less than the averaged internal photometric error for that star. The resulting deep-frame photometry was then merged with that determined using the short-exposure frames. The positions of each star was determined using the STARLINK package ASTROM and star positions from the UKST/COSMOS database, and as such are accurate to $\sim 0.2$ arcsec.

\section{Results}

The selection of candidate cluster members was made by placing theoretical isochrones on colour-magnitude diagrams and selecting stars from their positions with respect to the isochrones. The theoretical isochrones of D'Antona \& Mazzitelli (1994), and in particular those using Alexander, Rodgers \& Iglesias opacities with the Canuto \& Mazzetelli convection model, remain the most comprehensive for the low-mass stars with which we are concerned here. However, the calibrations used to transform the isochrones from the theoretical quantities of $T_{\text {eff }}$ 
and $\log \frac{L}{L_{0}}$ to the observed colours and magnitudes are not well defined.

For $4000 \mathrm{~K}<T_{\text {eff }}>3500 \mathrm{~K}$ we used temperature scales and bolometric corrections from Bessell (1995) and for $T>4000 \mathrm{~K}$, temperature scales and bolometric corrections of Kurucz computed by Wood \& Bessell (private communication) which are available via anonymous ftp from mso.anu.edu.au. For cooler stars Stauffer et al. (1995) have made comparisons of a $70 \mathrm{Myr}$ isochrone, using several different temperature scales and bolometric corrections, with known Pleiades members. The best agreement was achieved using temperature scales from Kirkpatrick et al. (1993) with bolometric corrections from Bessell (1991). Thus, for $T_{\text {eff }} \leq 3500 \mathrm{~K}$, we have used temperature scales from Kirkpatrick et al. (1993) with bolometric corrections from the more recent paper by Bessell (1995).

Given the uncertainty in the literature regarding the age of the cluster, ranging from $8 \mathrm{Myr}$ to $36 \mathrm{Myr}$, it was decided to use 10 and $40 \mathrm{Myr}$ isochrones as the limits for selection of cluster members. The isochrones were transformed to allow for a distance modulus of 6.0 and reddening of $E_{V-I}=0.044$ (Randich et al. 1995) and broadened to allow for the $0.2 \mathrm{mag}$ uncertainty in the distance modulus. These limits were further broadened to allow for the photometric errors. These errors for field IC 2602a are shown in Table 2.

We have also taken into account the effect of binarity on the location of stars with respect to the theoretical isochrones. The size of the effect depends of the composition of the binary. We assume that if the unresolved companion has a lower mass and hence redder colour, it will have the effect of shifting the position to a brighter and redder position in the colour-magnitude diagram. For a companion of equal mass, the increase in brightness corresponds to 0.75 mag. However, Dabrowski \& Beardsley (1977) have shown that the increase in magnitude in the case of some binaries is as large as $0.8 \mathrm{mag}$, so we have decreased the bright selection limit by $0.8 \mathrm{mag}$ to allow for the presence of binaries. We note that the sequence of existing members from Prosser et al. (1996) show a width of $\sim 1.5$ mag. Given the increase in photometric error introduced though undersampling we feel that our broader selection criteria are justified.

Primary candidate members of the cluster were those stars which were located between the selection limits in both $(V, V-I)$ and $(R, R-I)$ colour-magnitude diagrams. Stars were selected as secondary candidate members if they fell between the limits in one or other of the diagrams but not both. Closer inspection of the secondary candidate members reveals that many of them are very unlikely to be true cluster members, having colours that place them far from the selection limits in the non-selecting diagram.

A $V$ vs. $V-I$ colour-magnitude diagram for field IC 2602a can be seen in Fig. 2, for the second cluster field (IC 2602b) in Fig. 3 and for the "offset" field in Fig. 4.
Table 2. Photometric errors for field IC 2602a

\begin{tabular}{lrrr}
\hline \hline Magnitude range & $\operatorname{Err}(V)$ & $\operatorname{Err}(V-R)$ & $\operatorname{Err}(V-I)$ \\
\hline $12<V<13$ & 0.02 & 0.04 & 0.03 \\
$13<V<14$ & 0.03 & 0.06 & 0.04 \\
$14<V<15$ & 0.04 & 0.08 & 0.05 \\
$15<V<16$ & 0.05 & 0.10 & 0.06 \\
$16<V<17$ & 0.07 & 0.15 & 0.10 \\
$17<V<18$ & 0.08 & 0.17 & 0.12 \\
$18<V<19$ & 0.13 & 0.14 & 0.13 \\
$19<V<20$ & 0.13 & 0.14 & 0.13 \\
\hline
\end{tabular}

It is clear from the colour-magnitude diagrams that the number of stars increases dramatically for $V>18.5$ in field IC $2602 \mathrm{~b}$, due to an increased scatter from the main field population. In view of this increased level of contamination we will consider only the primary candidate members with $V \leq 18.5$. In field IC 2602a we detect 45 primary candidate members. In field IC $2602 \mathrm{~b}$ we detect 33 primary candidate members. The primary candidate cluster members are listed in Table $3^{1}$.

The results of the $\mathrm{H} \alpha$ data are shown in Fig. 5, as a plot of $R$ minus an instrumental $\mathrm{H} \alpha$ magnitude (with an arbitrary zero-point) versus $R$ for field IC 2602a. The figure also shows the position of our photometrically selected primary candidate members. Figure 6 shows a similar diagram for field IC $2602 \mathrm{~b}$.

Photometry of all the stars observed can be obtained electronically from the Armagh Observatory WWW server (http://star.arm.ac.uk/ dcf/ic2602.html) or by anonymous ftp upon request.

\section{Discussion}

\subsection{Background contamination}

Given the low galactic latitude of IC $2602(b=-4.9)$ it is to be expected that the stars selected using theoretical isochrones will be contaminated to a large extent by background stars. In order to gauge the scale of this contamination a similar selection process was applied to the photometry of the "offset" field. The selection process yielded 43 "primary candidate members" with $V<18.5$.

It should be noted that in using the offset field to gauge the background contamination we are assuming that the distribution of stars in it is representative of the background stars to be found in the cluster fields. This comparison suggests that nearly all of the stars selected are background stars. However, see the discussion in Sect. 6.2 for further comments on the location of the offset field.

\footnotetext{
1 Table 3 is only available in electronic form at the CDS via anonymous ftp to cdsarc.u-strasbg.fr (130.79.128.5) or via http://cdsweb.u-strasbg.fr/Abstract.html
} 


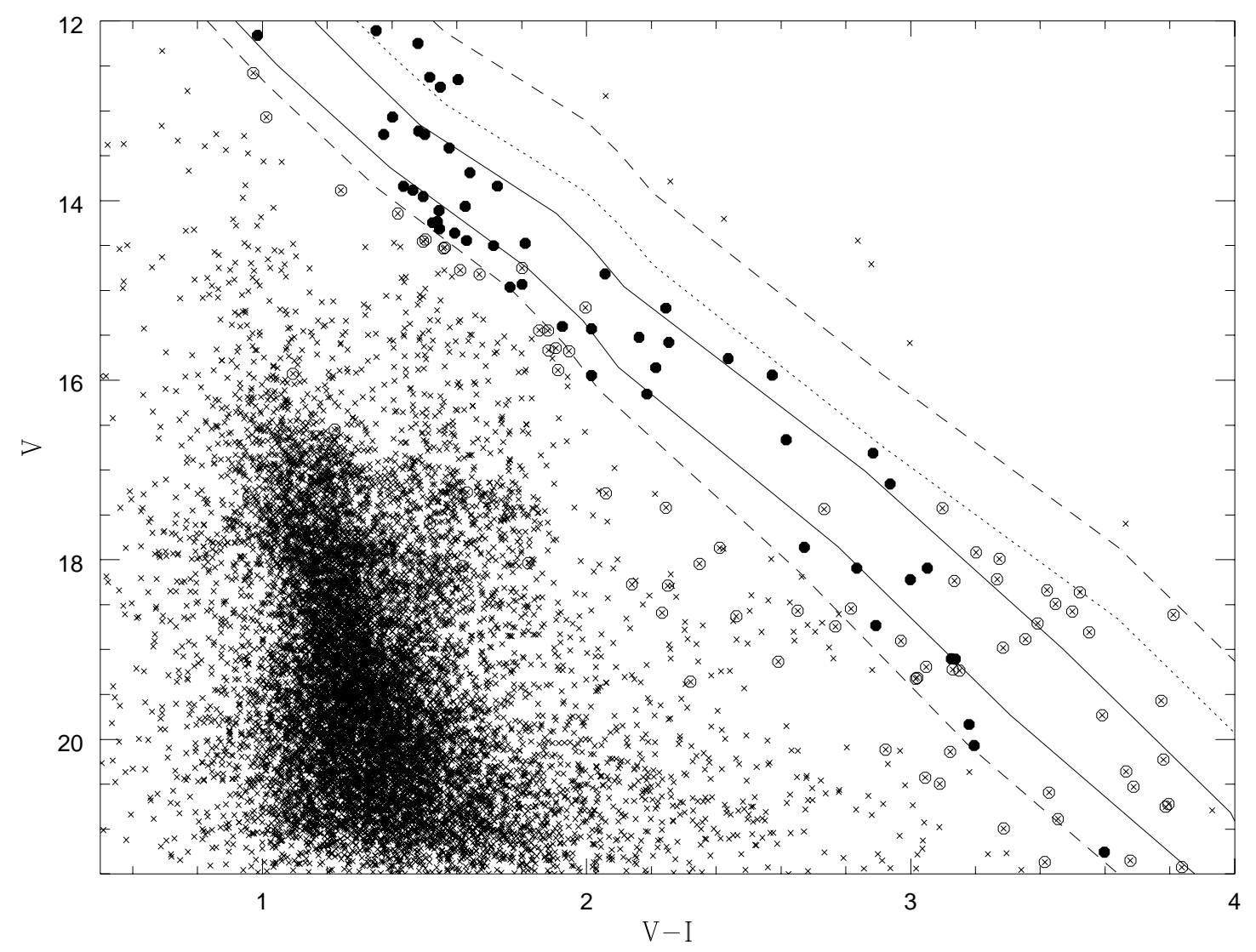

Fig. 2. A $V$ vs. $V-I$ colour magnitude diagram for the first cluster field showing the stars selected as primary (filled circles) and secondary (open circles) candidate cluster members. The solid lines are $10 \mathrm{Myr}$ and $40 \mathrm{Myr}$ isochrones, the dashed lines show the selection limits including all sources of uncertainty discussed in the text, and the dotted line shows the bright selection limit before any allowance was made for binarity

\subsection{Comparison with the results of Randich et al.}

In their study of IC 2602 using the ROSAT X-ray satellite, Randich et al (1995) detected 110 X-ray sources in an $11 \mathrm{deg}^{2}$ area, 68 of which they identify with at least one optical counterpart. 4 of these X-ray sources lie in our field IC 2602a: R64, R69, R76, R80. No detected X-ray sources lie in our second cluster field. One X-ray source, R25, lies in our offset field. Randich et al. identify 7 stars as possible optical counterparts to these X-ray sources (6 in field IC 2602a, 1 in our offset field) based on photometrically selected cluster members and additional bright stars located close to the X-ray position.

Table 4 shows the results of our photometry for the stars which Randich et al. indicate as optical counterparts. Photometry for the X-ray selected stars is taken from Prosser et al. (1996) where available. The X-ray source R80 is clearly identified with the bright star HD 308016 whose magnitude of $V=10.66$ places it beyond the bright limit of our photometry.

Comparing the remaining 6 stars, there are differences in both the $V$ magnitude and the $V-I$ colours between the two sets of photometry somewhat larger than the errors
Table 4. A comparison of our photometry with that listed in Randich et al. and Prosser et al. For details of stars with IDs "f1" or "of" refer to tables on the WWW site

\begin{tabular}{lrrrr}
\hline \hline ID & $V$ & $V-R$ & $V-I$ & Memb. \\
\hline R25 & 16.38 & - & 2.75 & $\mathrm{Y}$ \\
of-6078 & 16.15 & 1.00 & 2.67 & $\mathrm{Y}$ \\
\hline R64 & 16.53 & - & 2.51 & $\mathrm{Y} ?$ \\
F41 & 16.66 & 0.93 & 2.62 & $\mathrm{Y}$ \\
\hline R69A & 18.0 & - & 5.40 & $?$ \\
f1-514 & 16.71 & 2.08 & 5.12 & $\mathrm{~N}$ \\
\hline R69B & 14.12 & - & 2.36 & $\mathrm{~N}$ \\
f1-10 & 14.20 & 1.11 & 2.42 & $\mathrm{~N}$ \\
\hline R69C & 17.07 & - & 2.87 & $\mathrm{Y}$ \\
f1-738 & 17.16 & 1.04 & 2.94 & $\mathrm{Y} ?$ \\
\hline R76 & 16.81 & - & 2.97 & $\mathrm{Y} ?$ \\
F71 & 16.81 & 1.07 & 2.88 & $\mathrm{Y}$ \\
\hline & & & &
\end{tabular}

in Table 2. Given that Randich et al.'s objects are active stars, however, they are quite likely to be variable. van Leeuwen et al. (1987) have found variable stars in the 


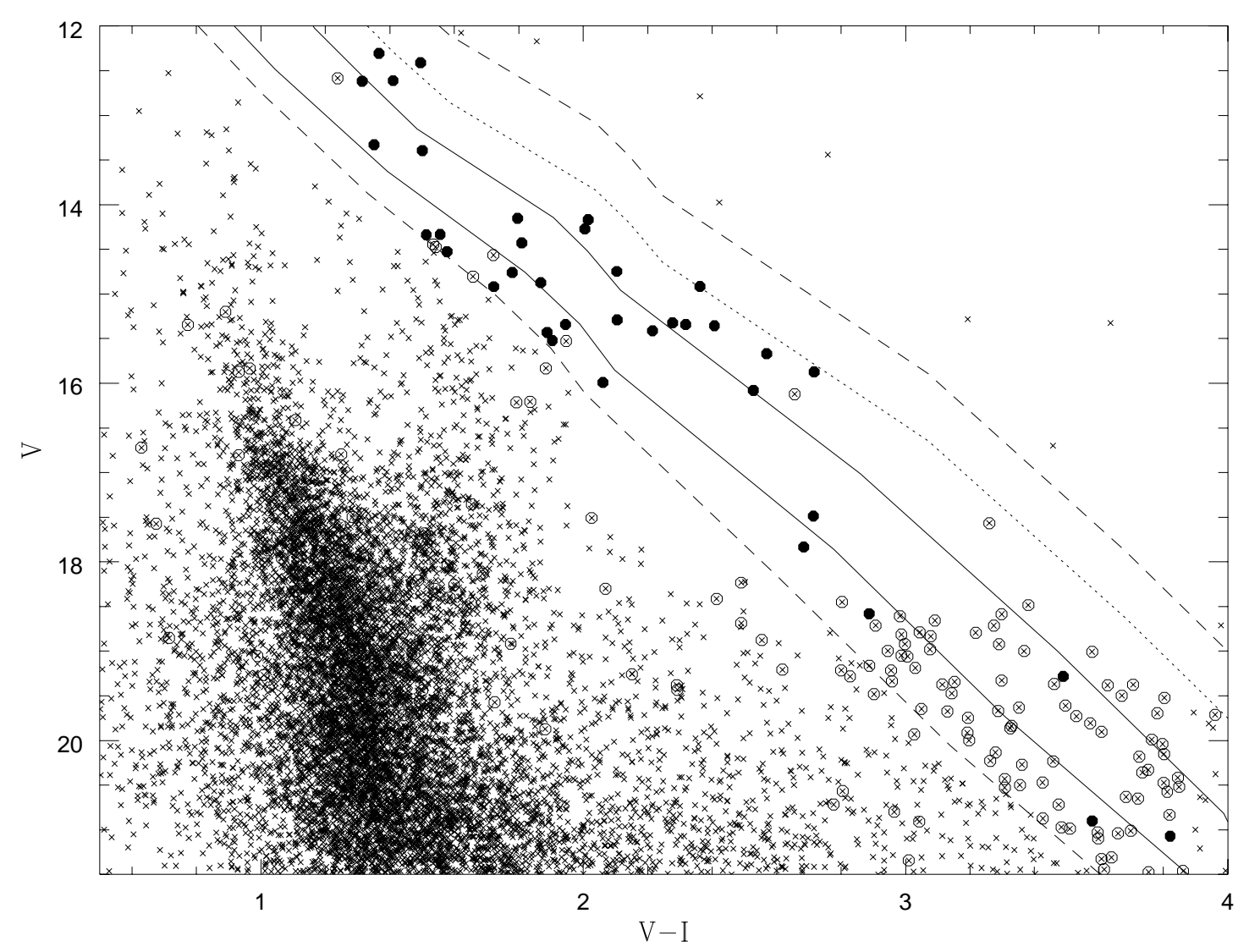

Fig. 3. A $V$ vs. $V-I$ colour magnitude diagram for the second cluster field with symbols as per Fig. 2

Pleiades with amplitudes of up to $\Delta V \sim 0.2$. Walter et al. (1992) have recorded variability on a naked T-Tauri star of amplitude $\Delta V \sim 0.5, \Delta(R-I) \sim 0.2$. Thus a part of the discrepancy at least might be reasonably attributed to such variability. Since the majority of Randich et al.'s photometry is unpublished we are unable to make a more detailed comparison between the photometric datasets at this point.

The positions of the detected X-ray sources in the region of IC 2602 are shown in Fig. 7. The figure also shows the positions of our observed fields. It should be noted that there are no X-ray sources in the the field IC $2602 \mathrm{~b}$ and that there are relatively few in the region surrounding it. This lack of X-ray sources is consistent with the lack of primary candidate members found from the photometry of that field. It should also be noted that the offset field contains one X-ray source, R25. Both our photometry and that of Randich et al. indicated that this star is a probable cluster member. If this star is a true cluster member then the offset field may be positioned too close to the cluster to give an accurate indication of the background contamination. If this is the case then we are over-estimating the level of background contamination as the "primary candidate members" will contain real cluster members as well as background stars which lie in the appropriate areas of the colour-magnitude diagrams.

\subsection{Comparison with the Pleiades}

The Pleiades, by virtue of its proximity, is one of the most extensively studied young (age $\sim 70 \mathrm{Myr}$ ) open clusters. Hambly \& Jameson (1991) have studied its massdistribution and luminosity function. We may use their results to independently examine the effects of background contamination on our data.

By taking the star numbers for the inner $0.6^{\circ}$ radius of the Pleiades as an approximate model for our region of IC 2602, and scaling to allow for the different distance moduli and angular extents of the two clusters, we should see roughly 22 cluster members in our cluster fields. Insofar as this comparison is valid, this suggests that the level of contamination is less than that suggested by the number of "primary candidate members" located in the offset field, assuming that the two clusters have similar star densities and mass functions. We have made no allowances for the differences in "richness" between the two clusters.

In summary, the evidence suggests that the level of contamination due to background stars lies somewhere between $50 \%$, as suggested by Pleiades mass functions, and $73 \%$ as suggested by comparing the "offset" field with field IC 2602a, although it is likely to be well below the latter figure, given that the "offset" field appears to be 


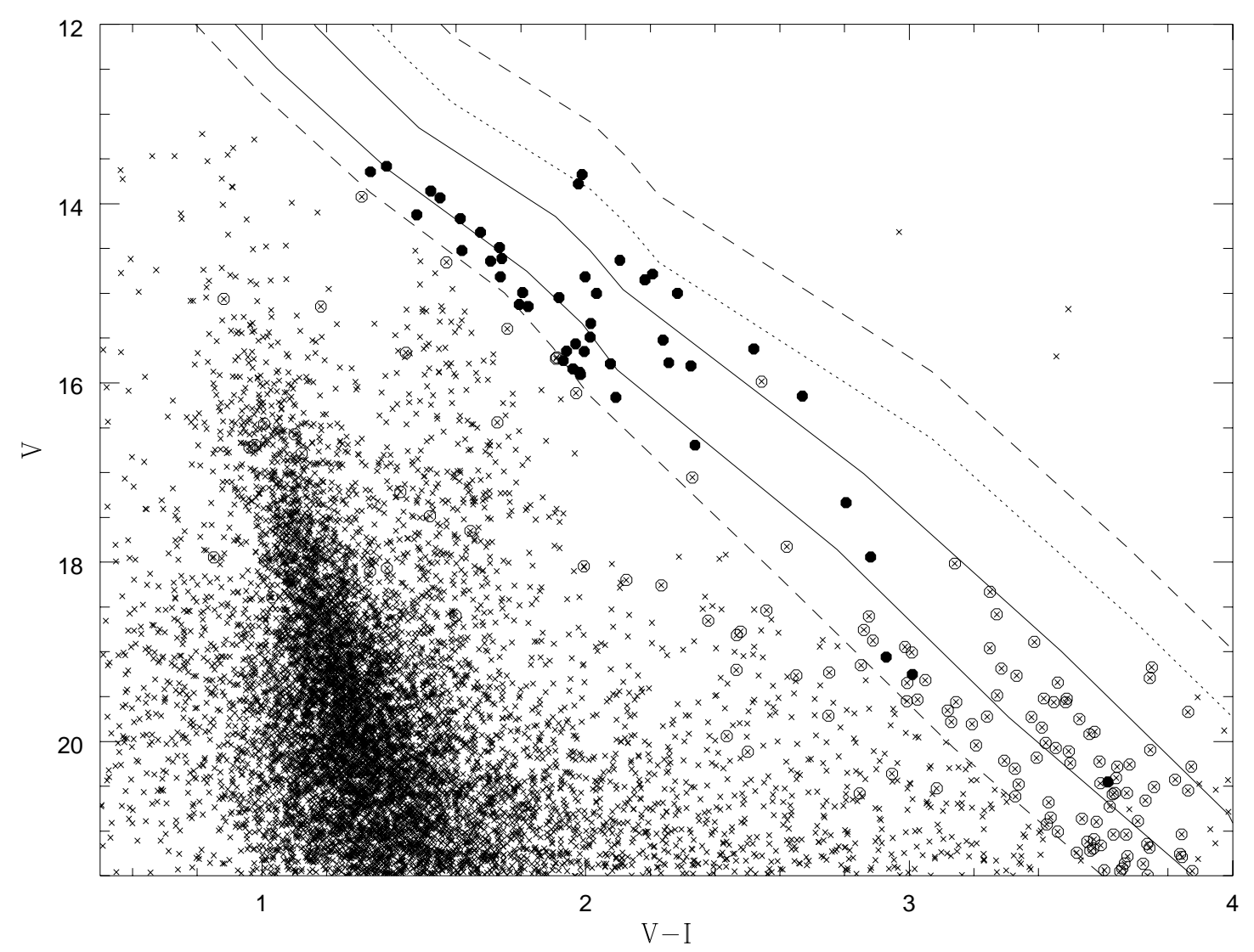

Fig. 4. A $V$ vs. $V-I$ colour magnitude diagram for the offset field with symbols as per Fig. 2

located within the cluster. Such levels of contamination might well be expected given the location of the cluster, and the broad selection limits used.

\subsection{Luminosity function}

In Fig. 8, which shows the distribution of primary candidate members with $V$ magnitude, several features are apparent. Firstly, for $12<V<14$ there is a clear excess of cluster members in field IC 2602a in comparison with cluster field IC 2602b and the offset field. This excess is not apparent in the range $14<V<16$ where an effectively similar number of primary candidate members was selected in each cluster field. A larger number of stars were selected in the offset field in this magnitude range. In each colour-manitude diagram there is an obvious field-giant branch with $1.4<V-I<1.9$. This may be a cause of contamination in the candidate list for $V<14.5$, but a reason why this should be worse in the offset field in comparison with the other two fields is unclear. There is a sharp falloff in stars in all fields in the range $16<V<19$. Because of the likely high level of field contamination, further dicussion of the cluster luminosity function is not appropriate at this time.

\section{5. $\mathrm{H} \alpha$ brightness}

Given that the $\mathrm{H} \alpha$ filter had a passband of $70 \AA$, that the equivalent width of $\mathrm{H} \alpha$ in an active late-M dwarf is $\sim 9 \AA$ and that the equivalent width in absorption for an inactive $\mathrm{M}$ dwarf is $\sim 1 \AA$, we would expect a $\mathrm{H} \alpha$ magnitude difference between active and inactive stars of $\sim 0.14 \mathrm{mag}$, assuming that cluster late-type stars are similar to solar neighbourhood M dwarfs. This magnitude difference is comparable to both the scatter in the plot and the errors in $R-\mathrm{H} \alpha$. Thus, we were unable to make any further selection on the basis of the $\mathrm{H} \alpha$ magnitudes.

We were able to determine $\mathrm{H} \alpha$ magnitudes for 20 of the 45 photometrically selected stars in cluster field IC 2602a (the rest being too bright). 13 of the 20 stars lie to the $\mathrm{H} \alpha$ bright side of the mean $R-\mathrm{H} \alpha$ level, 7 of these more than $3 \sigma$ from the mean level $(\sigma=\sim 4.2 \AA)$. Of the stars that lie below the mean, none are more than $1 \sigma$ from the mean. Similarly, for cluster field IC 2602b we have determined $\mathrm{H} \alpha$ magnitudes for 18 of the 33 primary candidate members. The scatter in the data is much larger $(\sigma=\sim 10 \AA)$, and so only two stars lie more than $1 \sigma$ from the mean, both in emission. The data for the primary candidate members are shown in Table 5.

For field IC 2602a the $\mathrm{H} \alpha$ data reinforces the likelyhood of a significant fraction of the photometrically 


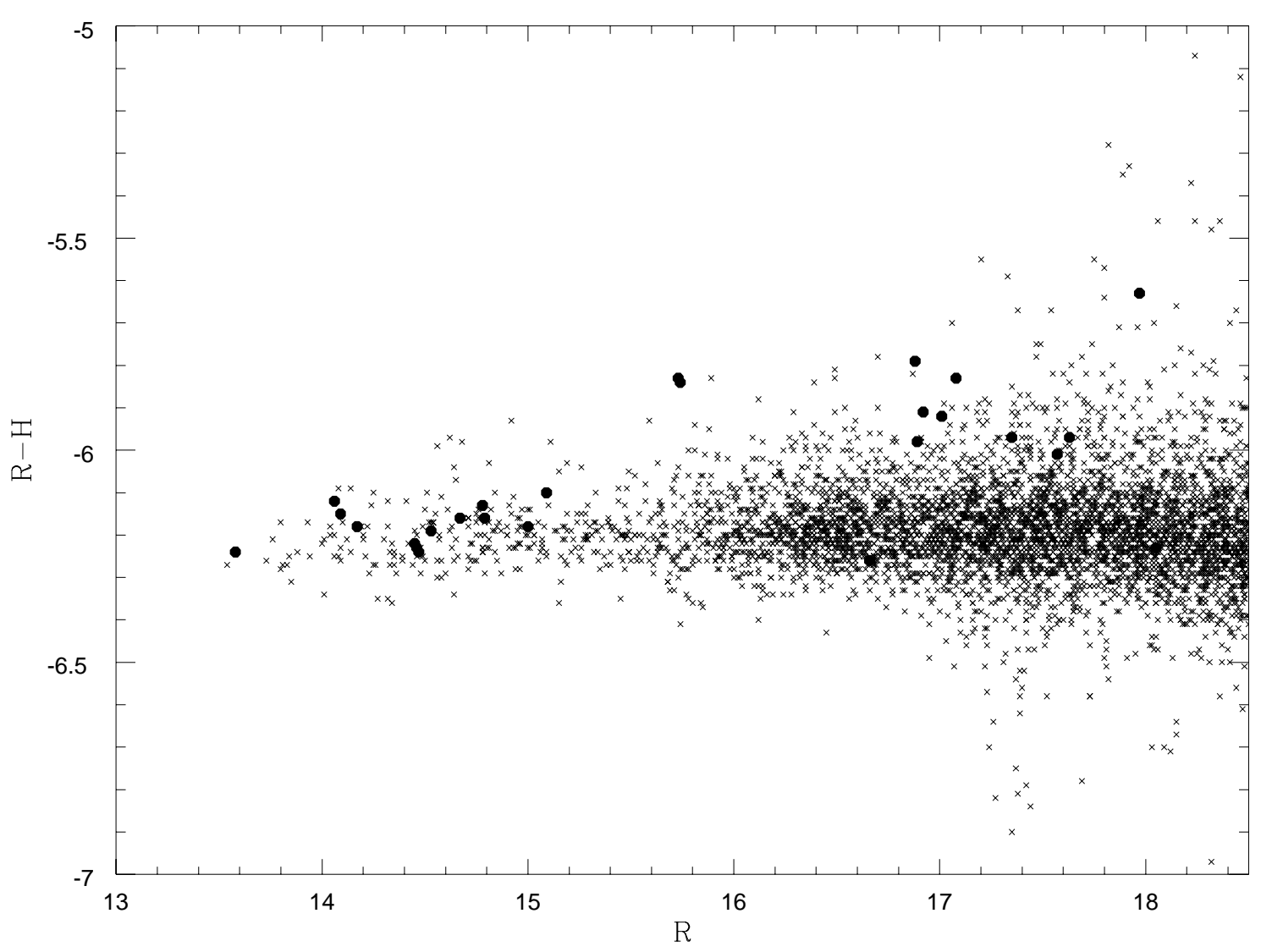

Fig. 5. Instrumental $r$ vs. instrumental $r-\mathrm{H} \alpha$ for cluster field IC 2602a. The solid dots are the photometrically selected primary candidate members for that field

selected star being true cluster members; $35 \%$ of those stars with $\mathrm{H} \alpha$ magnitudes being well in emission. For field IC $2602 \mathrm{~b}$ the results are also consistent with the reduced number of photometrically selected stars, and the lack of any X-ray detections in that field.

\section{Conclusions}

We have presented $V R I$ and narrow band $\mathrm{H} \alpha$ photometry of two $15 \times 15$ arcmin fields near the centre of the young open cluster IC 2602. The $V R I$ photometry was used to prepare colour-magnitude diagrams. Through the use of theoretical isochrones, we have identified 78 primary candidate cluster members. Using similar techniques on a nearby offset field, and through comparison with data on the Pleiades cluster from the literature, we have estimated the level of background contamination to be $\geq 50 \%$. The $\mathrm{H} \alpha$ data was also used to prepare colour-magnitude diagrams. The location of the photometrically selected stars on these diagrams reinforces the likelihood of their being cluster members. Independent methods, such as a proper motion study, or a study of radial velocities, are needed to confirm the selected stars as cluster members.

Acknowledgements. We would like to thank Dr. M. Read for providing the UKST/COSMOS positional data used to de- termine star coordinates. Research at Armagh Observatory is funded by a grant-in-aid from DENI. Data reduction was performed on the PPARC funded Northern Ireland STARLINK node. DCF acknowledges support of a studentship from Armagh Observatory. SLH was partially supported by NSF Young Investigator award AST94-57455. WRJR acknowledges financial assistance from the PPARC, grant number GR/J25352.

\section{References}

Abt H.A., Morgan W.W., 1972, ApJ 174, L131

Bessell M., 1991, AJ 101, 662

Bessell M.S., 1995, in "The Bottom of the Main Sequence" ESO Workshop, Tinney C. (ed.). ESO, Garching, p. 123

Braes L.L.E., 1962, Bull. Astron. Inst. Neth. 16, 297

D'Antona F., Mazzitelli I., 1994, ApJS 90, 467

Dabrowski J.P., Beardsley W.R., 1977, PASP 89, 225

Durney B., 1972, NASA SP-308, 282

Hambly N.C., Jameson R.F., 1991, MNRAS 249, 137

Hill G., Perry C.L., 1969, AJ 74, 1011

Kirkpatrick D., Kelly D., Rieke G., Liebert J., Allard F., Wehrse R., 1993, ApJ 402, 643

Kraft R.P., 1967, ApJ 150, 551

Landolt A.U., 1992, AJ 104, 340

Massey P., 1992, "A User's Guide to CCD Reductions with IRAF", NOAO Laboratory 


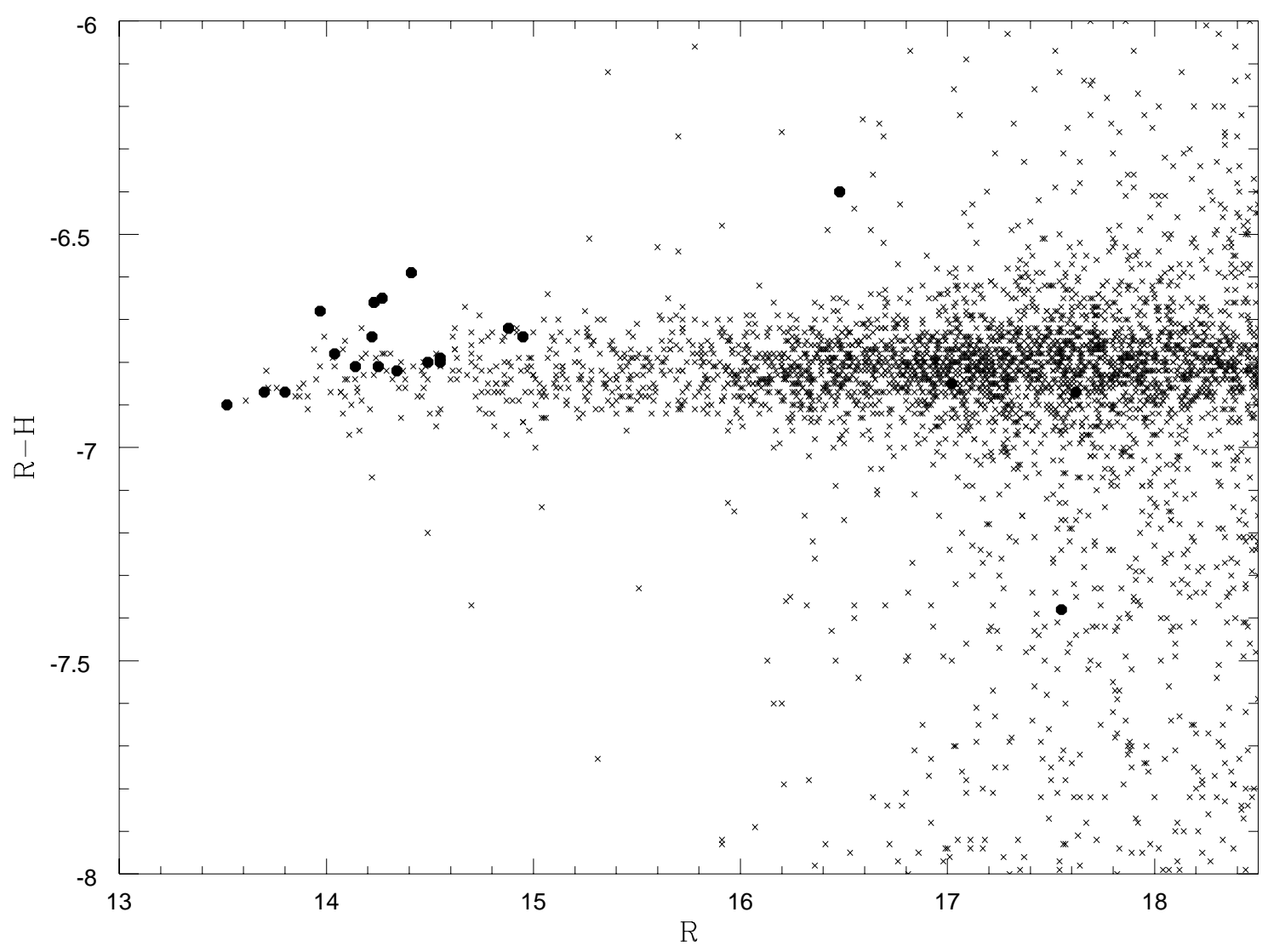

Fig. 6. Instrumental $r$ vs. instrumental $r-\mathrm{H} \alpha$ for cluster field IC $2602 \mathrm{~b}$. The solid dots are the photometrically selected primary candidate members for that field

Mermilliod J.C., 1981, A\&A 97, 235

Prosser C.P., Randich S., Stauffer J.R., 1996, AJ 112, 649

Randich S., Schmitt J.H.M.M., Prosser C.F., Stauffer J.R., 1995, A\&A 300, 134

Rolleston W.R.J., 1995, IAJ 22, 181

Skumanich A., 1972, ApJ 171, 565

Stauffer J.R., Hartmann L., Soderblom D.R., Burnham N., 1984, ApJ 280, 202

Stauffer J.R., Hartmann L., Burnham N., Jones B.F., 1985, ApJ 289, 247

Stauffer J.R., Hartman L., Latham D.W., 1987, ApJ 320, L51

Stauffer J., Hartmann L.W., Barrado D., 1995, ApJ 454, 910

Stetson P.B., 1987, PASP 99, 191

Stetson P.B., Davis L.E., Crabtree D.B., 1990, in "CCDs in Astronomy", ASP Conf. Ser. 8, 289

Tody D., 1986, IRAF User Manual, NOAO Laboratory van den Heuvel E.J., Conti P., 1971, Sci 171, 895

van Leeuwen F., Alphenaar P., Meys J.J.M., 1987, A\&AS 67, 483

Walter F.M., Vrba F.J., Brown A., 1991, in "Cool Stars, Stellar Systems, and the Sun", ASP Conf. Ser. 26, 340

Whiteoak J.B., 1961, MNRAS 123, 245

Wilson O.C., 1963, ApJ 138, 832
Table 5. $\mathrm{H} \alpha$ colour and equivalent widths for the primary candidate members

\begin{tabular}{lrrr|lrrr}
\hline \hline ID & $R$ & $R-\mathrm{H} \alpha$ & $W_{\mathrm{H} \alpha}$ & ID & $R$ & $R-\mathrm{H} \alpha$ & $W_{\mathrm{H} \alpha}$ \\
\hline F1 & 14.55 & -6.79 & -0.36 & F36 & 14.78 & -6.13 & 2.92 \\
F2 & 14.22 & -6.74 & 2.92 & F38 & 14.47 & -6.24 & -4.11 \\
F4 & 14.34 & -6.82 & -2.26 & F41 & 15.73 & -5.83 & 25.25 \\
F6 & 13.70 & -6.87 & -3.50 & F42 & 17.01 & -5.92 & 19.30 \\
F7 & 14.25 & -6.81 & -1.63 & F46 & 16.12 & -5.88 & 20.96 \\
F8 & 17.02 & -6.85 & -7.65 & F49 & 14.46 & -6.23 & -3.50 \\
F9 & 14.88 & -6.72 & 3.60 & F53 & 14.79 & -6.16 & 0.93 \\
F10 & 14.55 & -6.80 & -1.00 & F54 & 16.92 & -5.91 & 20.13 \\
F13 & 14.49 & -6.80 & -1.00 & F57 & 16.89 & -5.98 & 14.50 \\
F14 & 13.80 & -6.87 & -3.50 & F59 & 14.17 & -6.18 & -0.36 \\
F16 & 14.95 & -6.74 & 2.92 & F60 & 15.09 & -6.10 & 4.28 \\
F18 & 14.27 & -6.65 & 9.22 & F61 & 16.88 & -5.79 & 28.82 \\
F19 & 16.48 & -6.40 & 24.37 & F62 & 15.00 & -6.18 & -0.36 \\
F20 & 14.41 & -6.59 & 13.72 & F64 & 14.53 & -6.19 & -1.00 \\
F22 & 13.97 & -6.68 & 9.22 & F66 & 14.45 & -6.22 & -2.88 \\
F24 & 14.14 & -6.81 & -1.63 & F67 & 14.09 & -6.15 & 2.25 \\
F30 & 14.04 & -6.78 & 2.25 & F69 & 14.67 & -6.16 & 0.93 \\
F32 & 14.23 & -6.66 & 8.50 & F70 & 14.06 & -6.12 & 4.28 \\
F34 & 13.58 & -6.24 & -3.50 & F71 & 15.74 & -5.84 & 24.37 \\
\hline
\end{tabular}




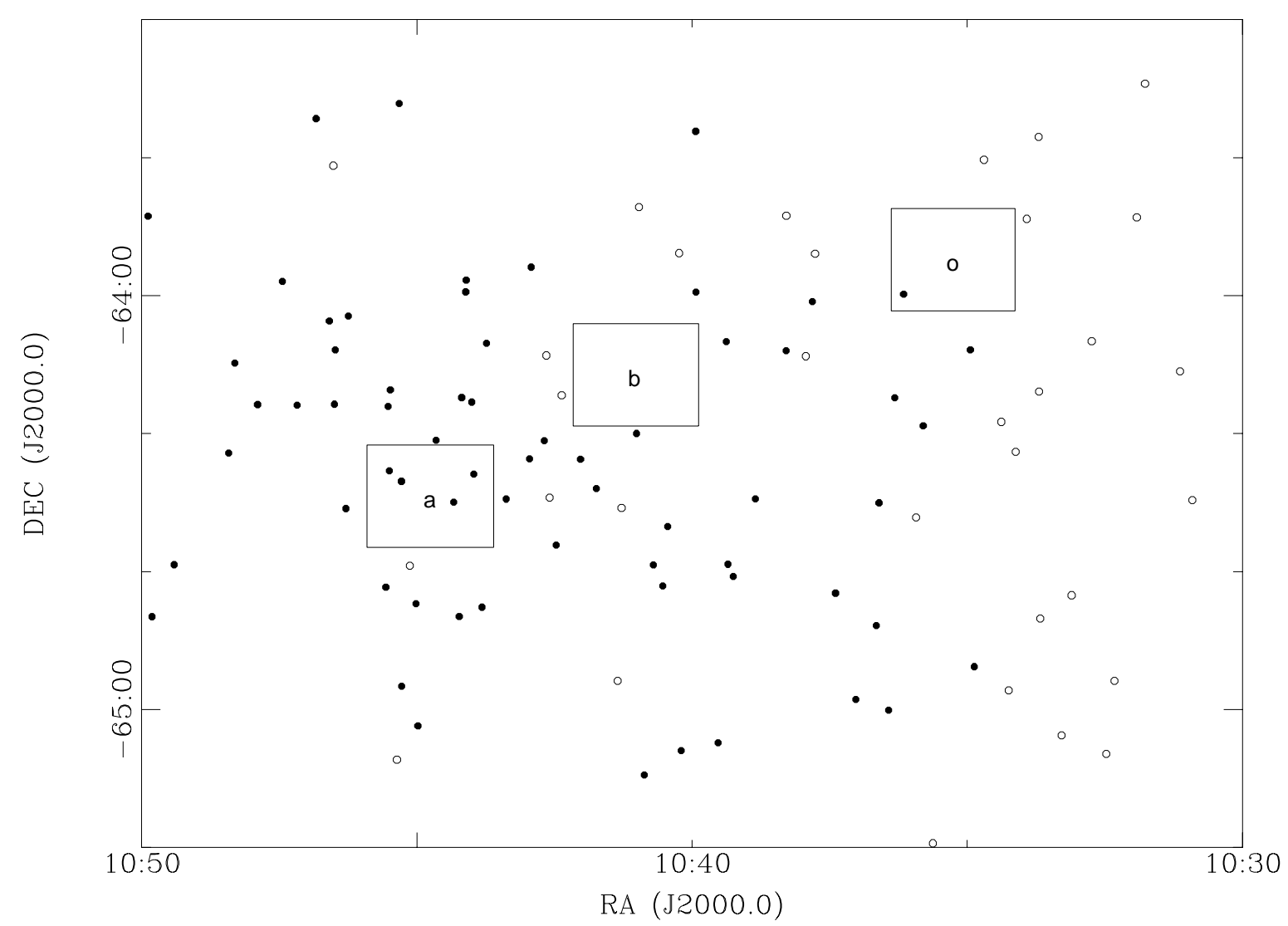

Fig. 7. The region of IC 2602 showing X-ray sources corresponding to photometric cluster members (filled circles) and non-members (open circles) from Randich et al. (1995)

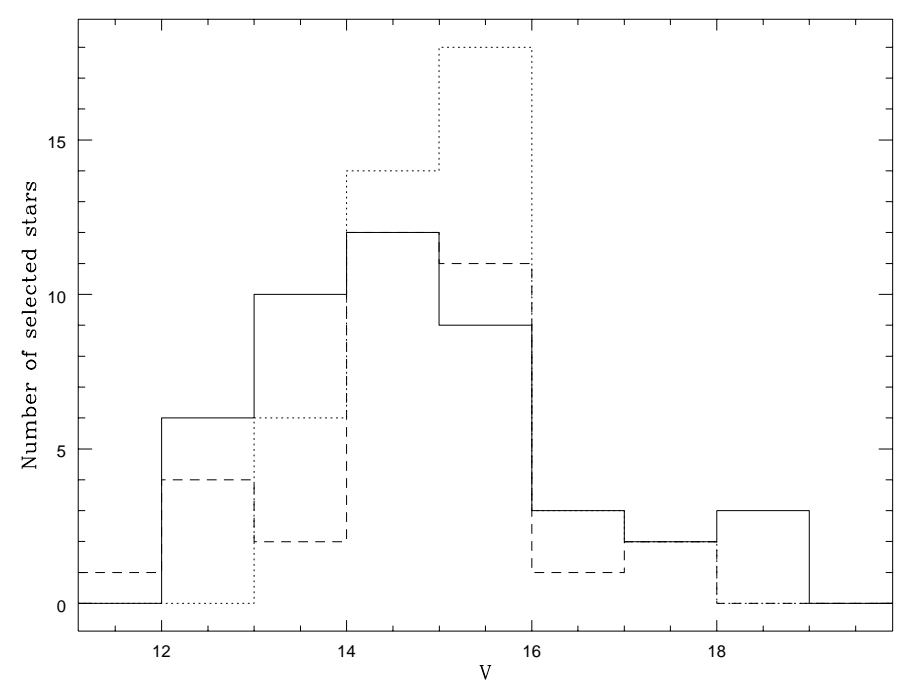

Fig. 8. The distribution of primary candidate members in IC 2602a (solid line), IC 2602b (dashed line) and the offset field (dotted line) with magnitude 